\title{
Improving proteinuria screening with mailed smartphone urinalysis testing in previously unscreened patients with hypertension: a randomized controlled trial
}

\author{
Julia Leddy ${ }^{1}$, Jamie A. Green ${ }^{1,2}$, Christina Yule ${ }^{2}$, Juliann Molecavage ${ }^{3}$, Josef Coresh ${ }^{4}$ and Alex R. Chang ${ }^{1,2^{*}}$ (I)
}

\begin{abstract}
Background: Proteinuria screening is recommended for patients with hypertension to screen for kidney disease and identify those at elevated risk for cardiovascular disease. However, screening rates among hypertensive patients are low. Home testing strategies may be useful in improving proteinuria screening adherence.

Methods: We conducted an individual-level, randomized trial at 55 primary care clinic sites in the Geisinger Health System to evaluate the effectiveness of a strategy using home smartphone urinalysis test (Dip.io) to complete proteinuria screening in previously unscreened non-diabetic patient portal users with hypertension. All patients received an educational letter and a standing urinalysis lab order, and then were randomized to control (usual care) or intervention. Intervention arm participants were invited to complete proteinuria screening with a mailed home smartphone urinalysis test. Co-primary outcomes were completion of proteinuria screening and number of albuminuria cases (albumin/creatinine ratio $[A C R] \geq 30 \mathrm{mg} / \mathrm{g}$ or protein/creatinine ratio $\geq 150 \mathrm{mg} / \mathrm{g}$ ) at the end of 3 months. We also evaluated patient satisfaction with the home test, and compliance with recommendations for patients with newly detected albuminuria.
\end{abstract}

Results: A total of 999 patients were randomized to intervention or control. Out of 499 patients assigned to the intervention arm, 253 were reached by phone, and 69/97 (71.1\%) consented patients completed the home test. Overall, the intervention increased proteinuria screening completion $(28.9 \%$ vs. $18.0 \% ; p<0.001)$ with no effect on the number of albuminuria cases (4 vs. 4) although only $6 / 57$ (10.5\%) patients with trace or $1+$ urine dipstick protein had a follow-up quantitative test. Among the 55 patients who completed a survey after the home test, $89 \%$ preferred testing at home rather than the physician's office.

Conclusions: A strategy using a home urinalysis smartphone test increased proteinuria screening rates in previously unscreened patients with hypertension and may be useful in increasing rates of proteinuria screening compliance. Future studies should evaluate use of home testing kits to screen for and confirm albuminuria, and determine whether improving early detection of kidney disease can improve future kidney health.

Trial registration: Clinical Trial Registry: NCT03470701 (First posted 3/20/2018)https://clinicaltrials.gov/ct2/show/ NCT03470701. This study was retrospectively registered.

Keywords: Albuminuria, Proteinuria, Screening, Smartphone, Urinalysis, Chronic kidney disease, CKD, mHealth, Home testing

\footnotetext{
* Correspondence: chaalex@gmail.com

${ }^{1}$ Geisinger Health System, Department of Nephrology, 100 N Academy Ave, Danville PA17822, USA

${ }^{2}$ Geisinger Health System, Kidney Health Research Institute, 100 N Academy

Ave, Danville PA17822, USA

Full list of author information is available at the end of the article
}

(c) The Author(s). 2019 Open Access This article is distributed under the terms of the Creative Commons Attribution 4.0 International License (http://creativecommons.org/licenses/by/4.0/), which permits unrestricted use, distribution, and reproduction in any medium, provided you give appropriate credit to the original author(s) and the source, provide a link to the Creative Commons license, and indicate if changes were made. The Creative Commons Public Domain Dedication waiver (http://creativecommons.org/publicdomain/zero/1.0/) applies to the data made available in this article, unless otherwise stated. 


\section{Background}

Presence of albuminuria or proteinuria is associated with increased risk for cardiovascular and kidney disease [1, 2]. While guidelines recommend screening for kidney disease using urinalysis dipstick for patients with hypertension (and urine albumin/creatinine ratio [ACR] for those with diabetes or chronic kidney disease [CKD]) $[3,4]$, screening remains suboptimal [5-8]. Top research priorities raised by patients with CKD include improving strategies of early kidney disease identification, which could allow implementation of interventions to slow progression of disease $[9,10]$. Presence of albuminuria or proteinuria can also be helpful in risk stratification to determine which patients may benefit from management with blood pressure medications, inhibition of the renin-angiotensin-aldosterone system, and statins [11].

One potential barrier to screening is that patients may not be able to provide a urine sample in the office. Extending testing to the home may be a novel strategy to improve proteinuria screening. For example, use of mailed fecal occult blood testing kits has been shown to double compliance with colorectal cancer screening [12, 13]. The objective of this randomized controlled trial was to evaluate the effectiveness of home urinalysis testing, using a smartphone urinalysis kit (Dip.io), among previously unscreened patients with hypertension receiving care at the Geisinger Health System.

\section{Methods}

This study was an individual-level, randomized trial that included 999 patients at 55 primary care clinic sites in the Geisinger Health System, a large integrated health system in rural Pennsylvania. Funding was provided by the National Kidney Foundation (NKF), and the Geisinger Institutional Review Board approved the protocol (2017-0516). Health care providers and pharmacists across the system received education about management of proteinuria as well as information about the research study in small group lectures.

\section{Study population}

We used electronic health record data to identify eligible patients with hypertension who had never undergone proteinuria screening by any method. Inclusion criteria included Geisinger primary care patients $\geq 18$ years of age with a hypertension diagnosis, blood pressure $\geq 130 / 80 \mathrm{mmHg}$ at last outpatient visit, and active patient portal use with a listed phone number and email address. Main exclusion criteria included diabetes, end-stage renal disease, and estimated glomerular filtration rate $($ eGFR) $<15 \mathrm{ml} / \mathrm{min} /$ $1.73 \mathrm{~m}^{2}$.

\section{Participant flow and intervention}

A total of 999 patients met the inclusion/exclusion criteria and received a mailed reminder to complete proteinuria screening, a National Kidney Foundation educational booklet about the importance of proteinuria screening, and a standing Geisinger lab urinalysis order March 19-21, 2018. Since proteinuria screening is standard-of-care for patients with hypertension, informed consent was not required for this initial contact. A computer program was then used to randomize patients $1: 1$ to the intervention or control arm, stratified by baseline eGFR $<60 \mathrm{ml} / \mathrm{min} / 1.73 \mathrm{~m}^{2}$ since CKD is also an indication for proteinuria screening.

Patients in the control arm received no intervention, whereas patients in the intervention arm received a notification letter about the home urine smartphone test approximately 2 weeks later, with an option to opt-out of further contact by the research team. This was followed by telephone calls by the Geisinger Survey unit, who made up to 7 attempts and left up to 3 voicemails. We also conducted weekly data pulls to identify and remove any patients from the call list who had already completed proteinuria screening at the Geisinger lab. Patients who provided consent were sent a text message link to download the dip.io app from the Apple Store or Google Play (Fig. 1). The testing kit, along with a project leaflet, were shipped by Healthy.io using a third-party fulfillment service. If participants had not completed the test within the next week, Healthy.io's call center contacted participants to validate receipt of kit and application and trouble-shoot any issues that may have prevented the participants from completing the test.

The dip.io test consists of a home test kit and a smartphone application. The kit consists of a standard 10 parameter urinalysis dipstick, a custom designed urine cup and a color-board, which enables accurate analysis in different lighting environments. To conduct the test, patients open the app, follow directions provided on-screen, collect urine in the provided container, dip the urinalysis dipstick (Acon Mission), place the dipstick on the color board, and then scan the dipstick and color board using the app. For this project, results were transmitted to a Health Insurance Portability and Accountability Act (HIPAA)-compliant website portal, which was accessed by the research team. The study team contacted patients with abnormal home test results, and ordered confirmation ACR testing for trace or greater urine protein on urinalysis, or repeat urinalysis testing at a Geisinger laboratory for other urinalysis abnormalities along with notification to the PCP. For patients with detected albuminuria, the study team sent a notification to the PCP with the following guideline-based recommendations: treat to office blood pressure $<130 / 80 \mathrm{mmHg}$, treat with ACEI or ARB and statin. Providers had the option of 


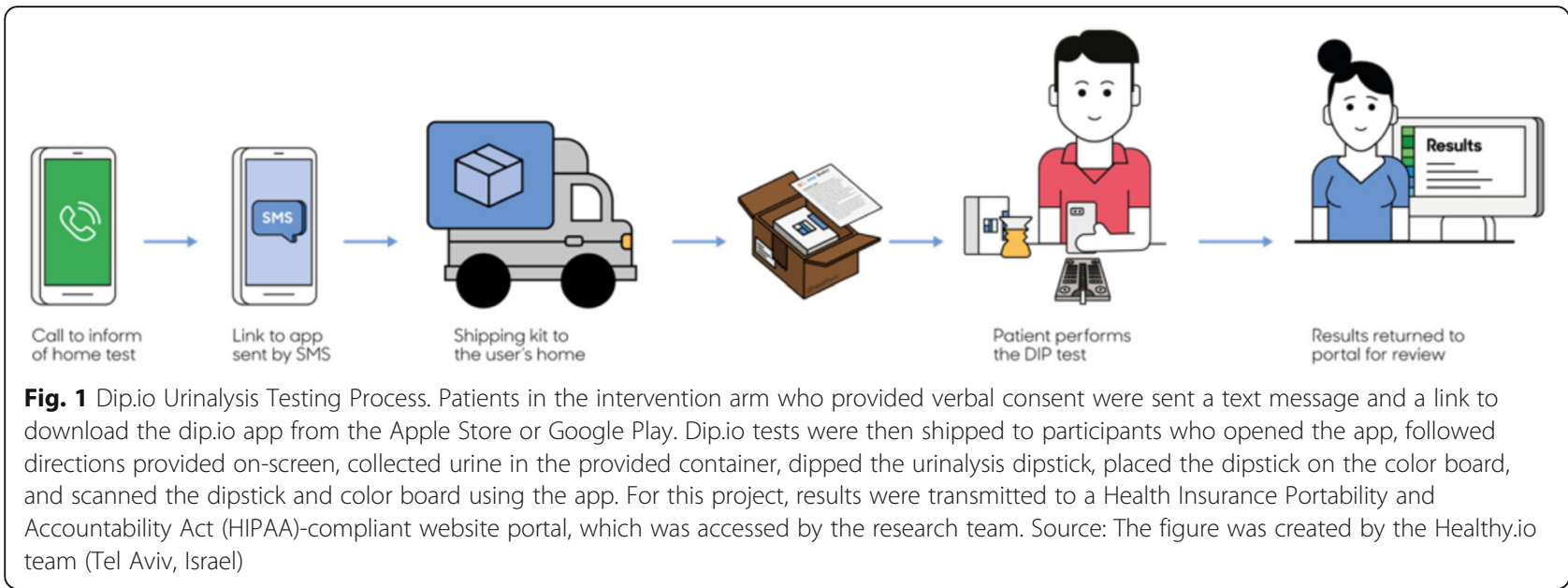

referral for further management by pharmacists, who received training in management of albuminuria and hypertension. PCPs were blinded to their patients' assignment with the exception that if an abnormal home test resulted, PCPs were later informed of the result. Final outcome data was assessed by a blinded member of the research team (JL).

\section{Outcomes}

Co-primary outcomes were completion of proteinuria screening by any method and number of quantified albuminuria cases (ACR $\geq 30 \mathrm{mg} / \mathrm{g}$ or protein/creatinine ratio $\geq 150 \mathrm{mg} / \mathrm{g})$ at the end of 3 months $(6 / 20 / 18)$. An exploratory outcome examined was the number of patients with trace or $1+$ urine protein tests at the end of 3 months.

\section{Analytic considerations}

With a sample size of 1000 total patients enrolled in the trial and assumptions of albuminuria prevalence of $20 \%$ [14], screening compliance rates of $40 \%$ in the intervention group and $10 \%$ in the control group, we estimated that we would have $>90 \%$ power to detect a difference in screening compliance between groups, and $>90 \%$ power to detect a difference in detected number of albuminuria cases.

We conducted intention-to-treat analyses and used logistic regression to examine the effects of the intervention on screening outcomes. Satisfaction with the home test was evaluated by a survey administered by smartphone after test completion. $P$ values $<0.05$ were considered statistically significant without correction for multiple comparisons, and analyses were completed using STATA version 15.1.

\section{Results}

Of the 999 qualifying patients in the trial, mean age was $50.5 \pm 11.8$ years, $40.6 \%$ were female, $93.8 \%$ were white, mean body mass index was $34.7 \pm 8.5 \mathrm{~kg} / \mathrm{m}^{2}$, mean systolic blood pressure was $139.8 \pm 14.0 \mathrm{mmHg}$, mean diastolic blood pressure was $86.2 \pm 9.2 \mathrm{mmHg}, 70.0 \%$ were on blood pressure medications, $80.3 \%$ had eGFR $\geq 60 \mathrm{ml} / \mathrm{min} /$ $1.73 \mathrm{~m}^{2}, 2.2 \%$ had eGFR $15-59 \mathrm{ml} / \mathrm{min} / 1.73 \mathrm{~m}^{2}$, and $17.5 \%$ had missing eGFR (Table 1). All participants were included in analyses.

\section{Participation in home testing in the intervention arm}

Of the 499 patients in the intervention arm, 7 (1.4\%) opted out of further contact after receiving the notification letter about the opportunity to use mailed smartphone urinalysis kit for proteinuria testing. Two weeks after the notification letter was sent, 40 patients in the intervention arm had already completed proteinuria screening at an outpatient Geisinger lab (Fig. 2). Of the

Table 1 Baseline Characteristics

\begin{tabular}{lll}
\hline & Intervention $(n=499)$ & Control $(n=500)$ \\
\hline Age $^{a}$ & $51.0(12.1)$ & $49.5(11.5)$ \\
White & $468(93.8)$ & $469(93.8)$ \\
Female & $201(40.3)$ & $203(40.6)$ \\
Ischemic heart disease & $29(5.8)$ & $19(3.8)$ \\
Cerebrovascular disease & $19(3.8)$ & $17(3.4)$ \\
On BP medications & $346(69.3)$ & $353(70.6)$ \\
BMl, kg/m² & $34.5(9.0)$ & $34.9(8.1)$ \\
SBP, mmHg & $139.5(14.2)$ & $140.1(13.9)$ \\
DBP, mmHg & $86.3(9.6)$ & $86.0(8.7)$ \\
eGFR, ml/min/1.73m ${ }^{2}$ & & \\
$\geq 60$ & $397(79.6)$ & $405(81.0)$ \\
$15-60$ & $13(2.6)$ & $9(1.8)$ \\
Missing & $89(17.8)$ & $86(17.2)$
\end{tabular}

Values for categorical variables are given as count (proportion); values for continuous variables are given as mean (SD)

Abbreviations: BP Blood pressure, BMI Body mass index, SBP Systolic blood pressure, DBP Diastolic blood pressure, eGFR Estimated glomerular filtration rate 


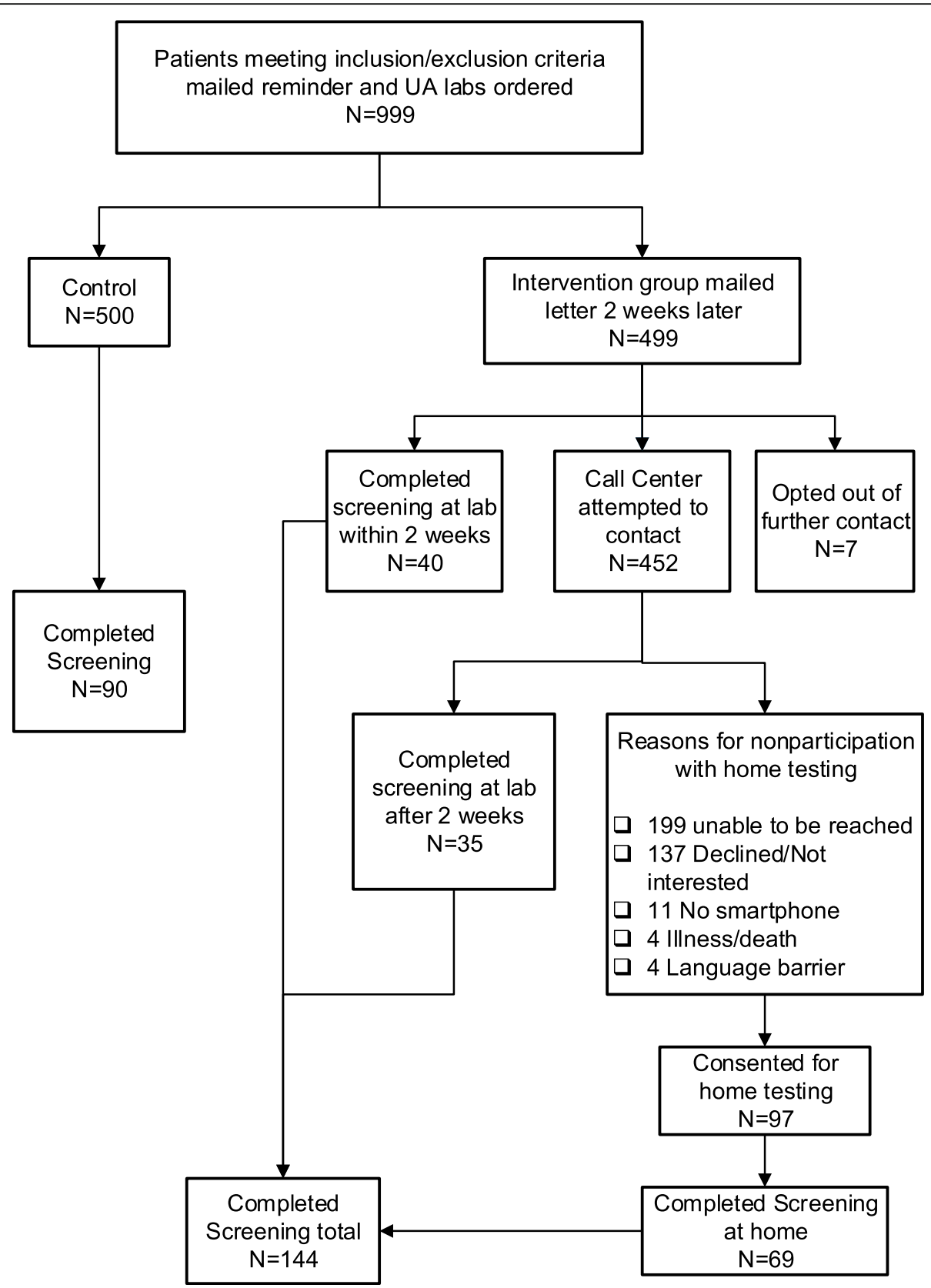

Fig. 2 Study Flow. A total of 999 patients met inclusion/exclusion criteria and were randomized; all were included in analyses. In the control arm, $90 / 500$ (18.0\%) completed proteinuria screening tests. In the intervention arm, a total of 144/499 (28.9\%) patients completed proteinuria screening tests, including 69 which were completed with the home testing kit

remaining 452 participants, the survey unit successfully reached 253 patients, and 35 participants completed proteinuria screening at an outpatient Geisinger lab. Reasons for declining to participate with home testing included lack of interest (137), no smartphone (11), illness/death (4), and language barrier (4). Out of $97 \mathrm{pa}-$ tients who consented for home testing, 69 (71.1\%) completed the home test, with 11 patients having trace protein and 1 patient having $1+$ protein.

\section{Effect of the intervention on screening outcomes}

Overall, the proportion of patients who completed proteinuria screening after 3 months was greater in the intervention arm than in the control arm $(28.9 \%$ vs. 18.0\%; odds ratio [OR] 1.85, 95\% confidence interval [CI]: 1.37-2.49) (Table 2). There was no significant difference between the intervention and control arms in the number of detected albuminuria cases ( 4 vs. 4; OR 1.00, 95\% CI: $0.25-4.03$ ) or an exploratory outcome of 
Table 2 Effect of the Intervention on Screening Outcomes

\begin{tabular}{|c|c|c|c|c|}
\hline & Intervention & Control & OR $(95 \% \mathrm{Cl})$ & $P$ value \\
\hline Screened by any method & $144(28.9 \%)$ & $90(18.0 \%)$ & $1.85(1.37,2.49)$ & $<0.001$ \\
\hline Quantified albuminuria cases (ACR $30+\mathrm{mg} / \mathrm{g}$ or PCR $150+\mathrm{mg} / \mathrm{g}$ ) & $4(0.8 \%)$ & $4(0.8 \%)$ & $1.00(0.25,4.03)$ & 1.00 \\
\hline Detected trace or $1+$ urine protein on dipstick & $34(6.8 \%)$ & $23(4.6 \%)$ & $1.52(0.88,2.61)$ & 0.13 \\
\hline
\end{tabular}

Abbreviations: ACR (albumin/creatinine ratio), OR (odds ratio)

detected trace or $1+$ urine dipstick protein (34 vs. 23; OR 1.52, 95\% CI: $0.88-2.61)$. Notably, only $3 / 23(13 \%)$ patients in the control group and 3/34 (9\%) patients in the intervention group with trace or $1+$ protein on urine dipstick had a quantitative test (ACR or protein/creatinine ratio) checked over the 3-month study period.

\section{Satisfaction with home testing}

Surveys were completed by $55 / 69$ (79.7\% response rate) patients after using the smartphone testing kit: $98 \%$ rated ease of use as "easy" or "very easy"; 93\% reported no problems with the device; $89 \%$ preferred testing at home rather than the physician's office; mean score for whether they would recommend home urine testing to a friend or colleague was $8.9(0-10,10=$ highest; net promoter score 62 [15]). No harms or unintended effects were noted by participants.

\section{Discussion}

In this multi-site, randomized controlled trial in a large, rural, integrated health system, we found that use of mailed smartphone urinalysis kits increased rates of albuminuria screening among previously unscreened patients with hypertension. While only $19 \%$ in the intervention arm received the home testing kit, $71 \%$ of those who received the kit completed testing. Those who completed home testing were highly satisfied and preferred testing at home vs. the lab, suggesting that home testing may be a useful strategy to improve compliance with proteinuria screening.

While our intervention improved screening, there was no difference in the number of detected albuminuria cases, confirmed by quantitative ACR or PCR testing. Several factors may have limited our ability to detect more albuminuria cases. Foremost, we required informed consent to send home testing kits to patients in the intervention arm. Thus, only $97 / 499$ (19.4\%) in the intervention arm provided consent to receive the home testing kit. In this type of low-risk intervention study where the goal is to improve adherence to screening, one might argue that a complete waiver of consent is desirable to maximize recruitment and generalizability of the intervention. Unfortunately, this study was conducted prior to the 2018 FDA approval of dip.io. Had this study been conducted after FDA approval, the IRB likely would have been able to grant a waiver of consent, and conducting this trial with a full waiver of consent would have allowed more patients in the intervention arm to receive the home testing kits and likely more screening completions. Second, we targeted non-diabetic, hypertensive patients, who had never received screening. This resulted in a study population that was at the healthier spectrum of at-risk patients who should be screened for albuminuria/proteinuria.

Another issue in our trial was that very few patients with trace or 1+ protein underwent confirmation testing by quantitative ACR or protein/creatinine ratio, over a relatively short follow-up period. Having learned how to use their smartphones to analyze the urine dipstick at home, it would have been intuitive to send an additional home testing kit to confirm whether ACR was elevated or not. Unfortunately, the Healthy.io semi-quantitative ACR home test was not available at the time of the trial, which could be an effective adjunct to confirm albuminuria at home. It seems likely that traveling to the laboratory for confirmation testing posed an additional barrier. Future studies should explore strategies where screening and confirmation of albuminuria is done solely using home testing kits.

Other studies have described similarly suboptimal rates of screening among patients at risk of CKD and even patients with eGFR $<60 \mathrm{ml} / \mathrm{min} / 1.73 \mathrm{~m}^{2}$. For example, in a study of stage 3-4 CKD patients receiving care from 2004 to 2008 in a group practice in eastern Massachusetts, only $30 \%$ had annual urine protein monitoring completed [16]. Even in an optimal setting where clinical decision support in the form of a CKD checklist embedded in the EHR was implemented in a prospective study, annual ACR measurement still was only able to be improved to 73\% [17]. Thus, use of home testing may have a role in further improving adherence to albuminuria/proteinuria screening.

There were several limitations in this proof-of-concept study. Due to the requirement of having a smartphone to test the urinalyses, we limited our mostly white, patient population to active patient portal users, and results may not be generalizable to non-portal users. While smartphone ownership has risen from 35\% in 2011 to $77 \%$ in 2018 [18], other screening strategies may also be needed to reach those without access to smartphones. It is possible that some dipstick protein results could be false negatives or false positives, and we lacked 
repeat testing on most abnormal dipstick protein results. Whether or not early detection of albuminuria can improve outcomes will require future large-scale studies with longer follow-up and consideration of strategies to increase patient engagement and provider adherence to clinical guidelines for albuminuria.

\section{Conclusions}

Our intervention using smartphone-based home testing was successful in increasing proteinuria screening rates in previously unscreened patients with hypertension and may be preferable for some patients. We also found that few patients with trace or $1+$ urine protein underwent follow-up ACR testing at the laboratory, limiting the effectiveness of a urine dipstick-first screening strategy. Future research directions should consider doing screening and confirmatory testing of ACR using home-testing modalities. Additional research is needed to determine whether improving adherence to albuminuria screening can improve patient-centered kidney and cardiovascular outcomes.

\section{Abbreviations \\ ACR: Albumin/creatinine ratio; Cl: confidence interval; CKD: chronic kidney disease; eGFR: estimated glomerular filtration rate; FDA: Federal Drug Administration; MTM: medication therapy management; NKF: National Kidney Foundation; OR: odds ratio; PI: principal investigator}

\section{Acknowledgments}

We thank Joel Schoppig and the Healthy.io (Tel Aviv, Israel) for creating and allowing us to use Fig. 1

\section{Funding}

This work was supported by a Research Grant of the National Kidney Foundation. A.C. also received support from the National Institutes of Health $(\mathrm{NIH}) /$ National Institute of Diabetes and Digestive and Kidney Diseases (NIDDK) grant K23 DK106515-01. Healthy.io provided mailed urinalysis testing in this trial free of charge. The funding source had no role in the design of this study, nor its execution, analyses, interpretation of the data, or decision to submit results.

\section{Availability of data and materials}

The datasets generated during this study are not publicly available, but are available from the corresponding author on reasonable request.

\section{Authors' contributions \\ Research idea and study design: ARC, CY, JAG, JM, JC; data acquisition: CY, JM; data analysis/interpretation/statistical analysis: JL, ARC; supervision or mentorship: ARC. All authors read and approved the final manuscript.}

\section{Ethics approval and consent to participate}

The Geisinger Institutional Review Board (IRB) approved the protocol (20170516), including the informed consent procedures which are as follows: Informed consent was obtained verbally for patients in the intervention arm to receive the home testing kit, which was not FDA-approved at the time of the research trial. Verbal consent was obtained as written consent would have required patients to come in person, limiting generalizability. Informed consent was waived for patients in the control arm as patients in the control received routine medical care. This study adheres to CONSORT guidelines (Supplemental Materials).

Consent for publication

Not applicable.

\section{Competing interests}

J.C. is on the Scientific Advisory Board for Healthy.io.

\section{Publisher's Note}

Springer Nature remains neutral with regard to jurisdictional claims in published maps and institutional affiliations.

\section{Author details}

${ }^{1}$ Geisinger Health System, Department of Nephrology, 100 N Academy Ave, Danville PA17822, USA. ${ }^{2}$ Geisinger Health System, Kidney Health Research Institute, 100 N Academy Ave, Danville PA17822, USA. ${ }^{3}$ Geisinger Health System, Ambulatory Medicine Institute, 100 N Academy Ave, Danville PA17822, USA. "Welch Center for Prevention, Epidemiology and Clinical Research, Johns Hopkins University, Baltimore, MD, USA.

Received: 5 December 2018 Accepted: 2 April 2019

Published online: 18 April 2019

\section{References}

1. Astor BC, Matsushita K, Gansevoort RT, van der Velde M, Woodward M, Levey AS, Jong PE, Coresh J, Chronic kidney disease prognosis C, Astor BC et al: lower estimated glomerular filtration rate and higher albuminuria are associated with mortality and end-stage renal disease. A collaborative metaxanalysis of kidney disease population cohorts. Kidney Int 2011, 79(12): $1331-1340$.

2. Matsushita K, Coresh J, Sang Y, Chalmers J, Fox C, Guallar E, Jafar T, Jassal SK, Landman GW, Muntner $P$, et al. Estimated glomerular filtration rate and albuminuria for prediction of cardiovascular outcomes: a collaborative metaanalysis of individual participant data. The lancetDiabetes \& endocrinology. 2015;3(7):514-25.

3. Whelton PK, Carey RM, Aronow WS, Casey DE Jr, Collins K, Dennison Himmelfarb C, DePalma SM, Gidding S, Jamerson KA, Jones DW, et al. ACC/AHA/AAPA/ABC/ACPM/AGS/APhA/ASH/ASPC/NMA/PCNA guideline for the prevention, detection, evaluation, and Management of High Blood Pressure in adults: a report of the American College of Cardiology/American Heart Association task force on clinical practice guidelines. Hypertension. 2017;2017.

4. Kidney Disease: Improving global outcomes (KDIGO) CKD work group. KDIGO 2012 clinical practice guideline for the evaluation and Management of Chronic Kidney Disease. Kidney inter., Suppl. 2013; 3: 1-150.

5. Litvin CB, Hyer JM, Ornstein SM. Use of clinical decision support to improve primary care identification and Management of Chronic Kidney Disease (CKD). Journal of the American Board of Family Medicine JABFM. 2016;29(5):604-12

6. Perkins RM, Chang AR, Wood KE, Coresh J, Matsushita K, Grams M. Incident chronic kidney disease: trends in management and outcomes. Clin Kidney J. 2016

7. Peralta CA, Frigaard M, Rubinsky AD, Rolon L, Lo L, Voora S, Seal K, Tuot D, Chao S, Lui K, et al. Implementation of a pragmatic randomized trial of screening for chronic kidney disease to improve care among non-diabetic hypertensive veterans. BMC Nephrol. 2017;18(1):132-017-0541-0546.

8. Stevens PE, O'Donoghue DJ, de Lusignan S, Van Vlymen J, Klebe B, Middleton R, Hague N, New J, Farmer CK. Chronic kidney disease management in the United Kingdom: NEOERICA project results. Kidney Int. 2007;72(1):92-9

9. Hemmelgarn BR, Pannu N, Ahmed SB, Elliott MJ, Tam-Tham H, Lillie E, Straus SE, Donald M, Barnieh L, Chong GC, et al. Determining the research priorities for patients with chronic kidney disease not on dialysis. Nephrol Dial Transplant. 2017;32(5):847-54.

10. Tong A, Crowe S, Chando S, Cass A, Chadban SJ, Chapman JR, Gallagher M, Hawley CM, Hill S, Howard K, et al. Research priorities in CKD: report of a National Workshop Conducted in Australia. Am J Kidney Dis. 2015;66(2):212-22

11. Stevens PE, Levin A, Kidney Disease: Improving global outcomes chronic kidney disease guideline development work group M: evaluation and management of chronic kidney disease: synopsis of the kidney disease: improving global outcomes 2012 clinical practice guideline. Ann Intern Med 2013, 158(11):825-830

12. Tinmouth J, Patel J, Austin PC, Baxter NN, Brouwers MC, Earle C, Levitt C, Lu $Y$, Mackinnon M, Paszat $L$, et al. Increasing participation in colorectal cancer 
screening: results from a cluster randomized trial of directly mailed gFOBT kits to previous nonresponders. Int J Cancer. 2015;136(6):E697-703.

13. Church TR, Yeazel MW, Jones RM, Kochevar LK, Watt GD, Mongin SJ, Cordes $\mathrm{JE}$, Engelhard D. A randomized trial of direct mailing of fecal occult blood tests to increase colorectal cancer screening. J Natl Cancer Inst. 2004;96(10): 770-80.

14. United States Renal Data System. USRDS annual data report: epidemiology of kidney disease in the United States: National Institutes of Health NloDaDaK; 2017

15. Reichheld FF. The one number you need to grow. Harv Bus Rev. 2003; 81(12):46-54, 124

16. Allen AS, Forman JP, Orav EJ, Bates DW, Denker BM, Sequist TD. Primary care management of chronic kidney disease. J Gen Intern Med. 2011;26(4): $386-92$

17. Mendu ML, Schneider LI, Aizer AA, Singh K, Leaf DE, Lee TH, Waikar SS: Implementation of a CKD checklist for primary care providers. Clin J Am Soc Nephrol : CJASN 2014, 9(9):1526-1535.

18. Mobile Fact Sheet [http://www.pewinternet.org/fact-sheet/mobile/].

Ready to submit your research? Choose BMC and benefit from:

- fast, convenient online submission

- thorough peer review by experienced researchers in your field

- rapid publication on acceptance

- support for research data, including large and complex data types

- gold Open Access which fosters wider collaboration and increased citations

- maximum visibility for your research: over $100 \mathrm{M}$ website views per year

At BMC, research is always in progress.

Learn more biomedcentral.com/submissions 